\title{
International Committee on Systematic Bacteriology Subcommittee on the Taxonomy of Leptospira
}

\author{
Minutes of Meetings, 1 and 2 July 1994, Prague, Czech Republic
}

Minute 1. Call to order. The meeting was called to order by the Secretary, R. Marshall, at 0930 on 1 July 1994. The opening consisted of a welcoming introduction, following which $\mathrm{R}$. Marshall was unanimously asked to act as Chairman for the meeting in the absence, because of illness, of the chairman, R. Yanagawa. W. Ellis accepted the job of meeting Secretary. Following this meeting there were additional open meetings at 1400 on 1 July 1994 and at 0900 on 2 July 1994. A closed meeting was held at 1300 on 2 July 1994.

Minute 2. Record of attendance. The members present were R. Marshall (Secretary), B. Cacciapuoti, M. Cinco, H. Dikken, W. Ellis, S. Faine, E. Kmety, R. Johnson, N. Stallman, W. Terpstra, and Y. Yanagihara. In addition to the members of the subcommittee, the following individuals attended one or more of the open meetings: B. Adler, P. Bakoss, G. Baranton, D. Brenner, S. Feresu, J. Hookey, Y. Kobayashi, H. Korver, G. Mazzonelli, J. Mazzonelli, P. Perolat, M. Saravi, and A. Schonberg.

Minute 3. Apologies for absence. Apologies for absence had been received from R. Yanagawa, A. Alexander, C. Bolin, M. Torten, Dai Boa-min, and A. Kaufmann.

Minute 4. Mark of respect. The subcommittee recorded with deepest regret the death of a member, K. Hovind-Hougen, and the deaths of former members C. D. Cox and J. van Riel.

Minute 5. Report on the activities of the subcommittee. A report of the subcommittee activities from 1991 to 1994 was read and accepted.

Minute 6. Minutes of the previous meeting. The minutes of the meeting held in Osaka, Japan, on 13 and 15 September 1990, which had been circulated previously, were accepted as a true record of the meeting.

Minute 7. Matters arising from the minutes of the previous meeting. S. Faine presented an update on the activities of the Genetic Working Group on Taxonomic Problems (see below).

Minute 8. WHO activity in international cooperation on leptospirosis control and research. W. Terpstra reported that T. Fujikura had retired from the World Health Organization (WHO) and that in discussions prior to the meeting it had been decided that $\mathrm{W}$. Terpstra would represent the WHO at the subcommittee meetings. W. Terpstra reported that at the International Conference on Leptospirosis Research which was held in Matsuyama, Japan, on 26 September 1990, a roundtable discussion chaired by T. Fujikura (WHO) and Y. Kobayashi had established working groups on (i) epidemiology and information exchange, (ii) diagnosis and related research, (iii) vaccine development and vaccinology, and (iv) clinical medicine and chemotherapy. These working groups were headed by S. Faine (Melbourne, Australia), W. Terpstra (Amsterdam, The Netherlands), R. Yanagawa (Sapporo, Japan), and Y. Kobayashi (Matsuyama, Japan), respectively. WHO employees had acted as group coordinators and facilitators in the development of the activities. Detailed reports on the working group activities were to be presented at a leptospirosis conference in Anzio, Italy, on 11 to 13 July 1994. S. Faine noted that the WHO could not provide financial support for the International Leptospirosis Information Exchange and for a revision of the Guidelines for the Control of Leptospirosis, which the WHO originally published in 1982 and which was in need of revision.
The subcommittee expressed general concern about the WHO's lack of support.

Minute 9. Usefulness of PCR-based strategies (MRSP and $P C R)$ for genospecies delimitation and molecular typing. P. Perolat presented a paper on the use of two PCR-based characterization methods for Leptospira reference strains and isolates. Arbitrarily primed PCR generates simple and reproducible fingerprints that can be used to identify leptospires at both the genospecies and serovar levels and for molecular epidemiology. Furthermore, a new PCR strategy, which is based on the study of mapped restriction site polymorphisms (MRSP) in PCR-amplified rrs (16S rRNA) and M (23S rRNA) eubacterial ribosomal genes, was developed and tested with Leptospira strains. The MRSP method allowed workers to group reference strains into 16 profiles which subdivided the genomic species defined in DNA relatedness studies. Both arbitrarily primed PCR and MRSP could be used routinely for identification of isolates at the species level, which is the first step in bacterial identification. By performing parsimony or genetic distance analyses with MRSP data, researchers determined the potential relationships among species with significant phylogenetic trees. A total of 48 serovar reference strains were typed at the genospecies and serovar levels by using MRSP and arbitrarily primed PCR, and the results were consistent with DNA-DNA hybridization and serotyping results; the only exceptions were the results obtained for Leptospira sp. serovars copenhageni and icterohaemorrhagiae, which were undistinguishable. Field isolates of Leptospira sp. serovar hardjo type hardjobovis were assigned to Leptospira borgpetersenii and were found to be very heterogeneous; conversely, isolates of Leptospira sp. type hardjoprajitno produced similar fingerprints and were included in Leptospira interrogans. Reference strains and isolates belonging to Leptospira sp. serogroups Grippotyphosa and Pomona belonged to five and four genospecies, respectively. Perolat concluded that PCR-based strategies (arbitrarily primed PCR and the MRSP method) appear to be simple and fast tools that can be used to study the genomic diversity of leptospires and have applications both in taxonomy and in epidemiology.

Minute 10. Molecular taxonomic study of leptospires. D. Brenner presented a paper on identification of leptospire serovars to the species level by DNA hybridization. Levels of DNA relatedness were determined by the hydroxyapatite method; $55^{\circ} \mathrm{C}$ was used as the optimal temperature for DNA reassociation, and $70^{\circ} \mathrm{C}$ was used as the stringent temperature for DNA reassociation. The genetic definition of a species (genomospecies or genomic species) used was a group of strains whose DNAs are $70 \%$ or more related at the optimal reassociation temperature, whose related DNA sequences contain $5 \%$ or fewer unpaired bases (divergence), and whose DNAs are $60 \%$ or more related at the stringent reassociation temperature. Approximately 300 strains, representing 255 serovars in the family Leptospiraceae, were tested. These strains were grouped into 12 named species, and there was a good possibility that there were up to four additional unnamed species. The geographic origins of the species varied significantly; for example, Leptospira santarosai and Leptospira noguchii were found al- 
most exclusively in the Americas, whereas Leptospira weilii was found almost exclusively in Europe and Asia. Individual species contained from 1 to 90 strains. It was found that frequently serovars within a given serogroup belonged to different species. There were also a number of instances in which one or more strains belonging to a single serovar were members of a species different from the species of the reference strain of that serovar. These results indicated that it should be simple to obtain species-specific probes for leptospires and also that serotyping does not correlate closely with species identification within these organisms.

Minute 11. Characterization of members of the Leptospiraceae by $16 S \mathrm{rDNA}$ restriction fragment length polymorphism. J. Hookey presented a paper on characterization of members of the Leptospiraceae on the basis of polymorphisms within and adjacent to the $r r n$ gene. Chromosomal DNAs from representative strains were digested with restriction endonucleases $B a m \mathrm{HI}$, $C l a \mathbf{I}$, and EcoRI, and Southern blots were hybridized with a biotinylated Escherichia coli $1.5-\mathrm{kb} 16 \mathrm{~S}$ ribosomal DNA (rDNA) probe. With the exception of a type strain (Leptospira interrogans serovar icterhaemorrhagiae strain RGA) and a neotype strain (Leptospira interrogans serovar icterohaemorrhagiae strain Ictero 1), all of the taxa examined could be differentiated from each other. Leptospira interrogans and Leptospira borgpetersenii reference strains were heterogeneous, whereas Leptonema illini and Leptospira parva (species incertae sedis) were distinct and separate. Strains of Leptospira biflexa produced different patterns. A porcine isolate was identified as a Leptospira interrogans serovar pomona type Pomona strain (J. Hookey, J. Gen. Microbiol. 139:1681-1690, 1993).

Minute 12. $16 S r D N A$ sequence analysis used to investigate the phylogeny of the Leptospiraceae and related bacteria. J. Hookey presented a paper on the natural evolution of the Leptospiraceae and related spirochetes as determined by an automated PCR cycle sequencing analysis of $16 \mathrm{~S}$ rDNA. The main points of the phylogeny were as follows: the serpulinas (Serpulina innocens and Serpulina hyodysenteriae) were closely related to each other and formed a line of descent that led to or from the Leptospiraceae; the Leptospiraceae comprised Leptospira parva, other Leptospira species, and Leptonema illini; on the basis of the results of this analysis and related genetic analyses there were sufficient data to propose a new genus, the genus "Turneria," for Leptospira parva; the pathogenic and saprophytic leptospires were separated from each other, and Leptospira inadai occupied an intermediate position between the two groups; the pathogens belonged to three groups, with group 1 represented by Leptospira interrogans and Leptospira kirschneri, group 2 represented by Leptospira weilli, Leptospira borgpetersenii, and Leptospira santarosai, and group 3 represented by Leptospira noguchii and Leptospira meyeri; the saprophytic species Leptospira biflexa and Leptospira wolbachii were similar to each other, yet these freshwater organisms were evolutionarily distant from the marine isolate Leptospira biflexa serovar ancona strain Ancona Porto; and the available 16S rDNA sequence data exhibited good correlations at and above a level of sequence similarity of $97 \%$ with species identified on the basis of DNA-DNA homology data (J. Hookey, J. Bryden, and L. Gatehouse, J. Gen. Microbiol. 139:2585-2590, 1993).

Minute 13. PFGE of leptospiral NotI-restricted DNA: molecular support for the serovar concept and an alternative method for serovar identification. G. Baranton described a study in which he analyzed 170 serovar reference strains by pulsed-field gel electrophoresis (PFGE) after their genomic DNAs had been digested with restriction enzyme NotI. Members of four groups of reference strains, including members of serovars lora, muenchen, jalna, and bratislava of the Australis serogroup, members of serovars castellonis and arborea of the Ballum serogroup, members of serovars losbanos and bataviae of serogroup Bataviae, and members of serovar copenhageni (strain M20) and serovar icterohaemorrhagiae (strain $\left.\mathrm{RGA}^{\mathrm{T}}\right)(\mathrm{T}=$ type strain), produced similar intragroup profiles when they were restricted with NotI, but members of the first two groups could be identified when their DNAs were restricted with SgrA1, while members of the last two groups could not be distinguished. As workers have found with other DNA fingerprinting methods, the serogroup concept was not supported by PFGE typing data. A total of 123 field isolates were subsequently typed by PFGE; the identities of $117(97 \%)$ of these isolates as determined by PFGE concurred with their identities as determined by serotyping.

Minute 14. Taxonomy of the nonpathogens. M. Cinco presented a paper in which she described phylogenetic studies of saprophytic strains based on 16S rRNA gene sequencing. The results of this study did not correlate with DNA-DNA hybridization results. Cinco concluded that DNA-DNA hybridization results should be applied to the taxonomy of saprophytes in the same way that they have been applied to the taxonomy of parasitic strains.

Minute 15. MAbs for easy and rapid typing. H. Korver presented a paper in which he reviewed the status of serotyping with batteries of monoclonal antibodies (MAbs) directed to relevant epitopes. MAbs are easy-to-use tools for quick identification of most leptospiral isolates, quality control, and tracing of mislabelled (diagnostic and vaccine) strains. MAbs allow identification of many isolates to the serovar level or even to the subserovar level. Most isolates submitted to reference laboratories for typing belong to a limited group containing approximately 30 reference strains. Now, more than one-half (a total of 122) of the recognized serovars can be identified by MAbs. Many nonreference laboratories have been equipped with panels of MAbs that allow quick and easy typing of most isolates. Panels of MAbs may provide characteristic antigenic profiles of leptospires even when serovar designation is not possible because of blurred boundaries between different but antigenically very similar leptospires (e.g., members of the Pomona and Grippotyphosa serogroups).

All MAbs are available to interested workers who want to use MAbs for typing or for research on typing. A booklet containing lists of MAbs and antigenic profiles was distributed.

Minute 16. Classification of strains belonging to the family Leptospiraceae by a gene-encoded character, the fatty acid profile. B. Cacciapuoti presented a paper in which he updated his previous work on the investigation of members of the Leptospiraceae by gas-liquid chromatography of fatty acid methyl ester (FAME) derivatives prepared from washed leptospires. Interstrain differences in the FAME profiles were quantified by performing a linear regression analysis in which the FAME profiles of pairs of strains were compared, and the results were expressed as correlation coefficients. Leptospiral strains could be differentiated into 17 FAME relatedness groups. In each group, a strain was selected as a FAME reference strain which could be used for chemotaxonomic classification of strains.

Minute 17. Species. The subcommittee recommended adoption of the species definition based on a level of DNA-DNA homology of at least $70 \%$ (L. G. Wayne et al., Int. J. Syst. Bacteriol. 37:463-464, 1987) and $\leq 5 \%$ divergence in DNA relatedness.

Minute 18. Serovars. The subcommittee recommended that taxonomy at the subspecific level should be based on serovars, as defined by H. Dikken and E. Kmety (Methods Microbiol. 11:259-307, 1978), but other valid methods can be used for identification. Such methods include analysis with MAbs or 
factor sera, analysis in which restriction fragment length polymorphism or rRNA gene restriction fragment patterns are used, and PFGE analysis.

Minute 19. Types. The subcommittee recommended that the term type should be used to indicate strain differences at the subserovar level.

Minute 20. Reference functions. The subcommittee recognized that the leptospirosis units at the following laboratories fulfil a reference function: Koninklijk Instituut voor de Tropen, Amsterdam, The Netherlands (W. Terpstra); Centers for Disease Control and Prevention, Atlanta, Ga. (A. Kaufmann); National Animal Disease Center, Ames, Iowa (C. Bolin); Pasteur Institute, Paris, France (G. Baranton); Public Health Laboratory Service, Hereford, United Kingdom; Veterinary Sciences Division, Belfast, United Kingdom; (W. Ellis); Queensland Health Laboratory of Microbiology and Pathology, Brisbane, Australia (L. Smythe); Istituto Superiore di Sanita, Rome, Italy (B. Cacciapuoti); University of Trieste, Trieste, Italy (M. Cinco); Gamaleya Institute of Epidemiology, Moscow, Russia (Y. Ananyina); Instituto Nacional de Tecnologia Agropecuaria, Buenos Aires, Argentina (M. Saravi and G. Mazzonelli); Komensky University, Bratislava, Slovakia (P. Bakoss); and National Institute for the Control of Pharmaceutical and Biological Products, Beijing, People's Republic of China (Qin Jingcai).

Minute 21. Reference laboratories. The subcommittee recommended that new pathogenic serovars should be verified at one of the following laboratories: Koninklijk Instituut voor de Tropen, Amsterdam, The Netherlands; Pasteur Institute, Paris, France; Komensky University, Bratislava, Slovakia; Queens- land Health Laboratory of Microbiology and Pathology, Brisbane, Australia; or Gamaleya Institute of Epidemiology, Moscow, Russia. New saprophytic serovars should be verified at the University of Trieste, Trieste, Italy.

Minute 22. Changes in membership and officers. W. Terpstra was elected Chairman, and W. Ellis was elected Secretary. B. Adler, P. Bakoss, C. Bolin, G. Baranton, and L. Smythe were elected members. The list of observers and collaborators was reviewed. M. Saravi, L. Ciceroni, M. Fukunaga, S. Feresu, and H. Korver were elected observers. G. Mazzonelli, Shi Man Hua, and R. Zuerner were elected collaborators. R. Yanagawa, A. Alexander, J. Coghlan, H. Dikken, S. Faine, E. Kmety, R. Johnson, N. Stallman, and M. Torten were elected honorary members.

Minute 23. Expression of appreciation. On behalf of the subcommittee, $\mathrm{W}$. Terpstra thanked the following retiring members for the service that they had given to the subcommittee: $R$. Yanagawa, H. Dikken, S. Faine, E. Kmety, R. Johnson, R. Marshall, N. Stallman, and M. Torten.

Minute 24. Current membership. The current members of the subcommittee are W. Terpstra (Chairman), Amsterdam, The Netherlands; W. Ellis (Secretary), Belfast, United Kingdom; B. Adler, Melbourne, Australia; Y. Ananjina, Moscow, Russia; C. Bolin, Ames, Iowa; P. Bakoss, Bratislava, Slovakia; G. Baranton, Paris, France; B. Cacciapuoti, Rome, Italy; L. Smythe, Brisbane, Australia; and Y. Yanagihara, Shizuoka-ken, Japan.

Minute 25. Adjournment. The final session was adjourned by the Chairman at 1630 on 2 July 1994.

W. A. Ellis, Secretary 